\title{
Structural and Biochemical Studies Reveal a Putative FtsZ Recognition Site on the Z-ring Stabilizer ZapD
}

\author{
Hwajung Choi ${ }^{1}$, Kyungjin Min ${ }^{1}$, Bunzo Mikami ${ }^{2}$, Hye-Jin Yoon ${ }^{1}$, and Hyung Ho Lee ${ }^{1, *}$
}

FtsZ, a tubulin homologue, is an essential protein of the Zring assembly in bacterial cell division. It consists of two domains, the $\mathrm{N}$-terminal and $\mathrm{C}$-terminal core domains, and has a conserved $\mathrm{C}$-terminal tail region. Lateral interactions between FtsZ protofilaments and several Z-ring associated proteins (Zaps) are necessary for modulating Z-ring formation. ZapD, one of the positive regulators of Z-ring assembly, directly binds to the C-terminal tail of FtsZ and promotes stable Z-ring formation during cytokinesis. To gain structural and functional insights into how ZapD interacts with the C-terminal tail of FtsZ, we solved two crystal structures of ZapD proteins from Salmonella typhimurium (StZapD) and Escherichia coli (EcZapD) at a 2.6 and 3.1 $\AA$ À resolution, respectively. Several conserved residues are clustered on the concave sides of the StZapD and EcZapD dimers, the suggested FtsZ binding site. Modeled structures of EcZapD-EcFtsZ and subsequent binding studies using bio-layer interferometry also identified the EcFtsZ binding site on EcZapD. The structural insights and the results of bio-layer interferometry assays suggest that the two FtsZ binding sites of ZapD dimer might be responsible for the binding of ZapD dimer to two protofilaments to hold them together.

\section{INTRODUCTION}

In bacterial cell division, cytokinesis is performed by a multiprotein complex called the divisome, which is composed of at least nine proteins (FtsA, Ftsl, FtsK, FtsL, FtsN, FtsQ, FtsW, FtsZ, and ZipA) (Margolin, 2000). FtsZ, a tubulin homolog, has a significant role in initiating divisome assembly by forming an organized polymer called the FtsZ ring (Z-ring), which then recruits other division proteins (Pichoff and Lutkenhaus, 2002).

${ }^{1}$ Department of Chemistry, College of Natural Sciences, Seoul National University, Seoul 08826, Korea, ${ }^{2}$ Laboratory of Quality Design and Exploitation, Division of Agronomy and Horticultural Science, Graduate School of Agriculture, Kyoto University, Kyoto 611-0011, Japan

*Correspondence: hyungholee @snu.ac.kr

Received 31 August, 2016; revised 25 October, 2016; accepted 26 October, 2016; published online 18 November, 2016

Keywords: cell division, cytokinesis, FtsZ, ZapD
The Z-ring can be mobilized by FtsA or ZipA to the membrane and is located midcell (Adams and Errington, 2009). FtsA interacts with the C-terminal domain of FtsZ, which enhances initial assembly and stability of the Z-ring (Adams and Errington, 2009; Lowe and van den Ent, 2001), while ZipA, a bitopic integral inner membrane protein, interacts with the C-terminal tail of FtsZ and anchors the complex to the membrane (Hale and de Boer, 1997; Ma et al., 1997). After adhering the Z-ring to the membrane, other division proteins, Ftsl, FtsK, FtsW, FtsQ, FtsL, and $\mathrm{FtsB}$, form the premature divisome. Then, the divisome matures through the adhesion of several proteins (PBP1B, LpoB, MltA, and EnvC) and binds to peptidoglycans and the outer membrane (Egan and Vollmer, 2013). The complete matured divisome is constricted by bending force and depolymerization of FtsZ filaments (Osawa et al., 2009).

FtsZ is highly conserved in most bacteria such as eubacteria and archaea, and is also seen in chloroplasts and some primitive mitochondria (Erickson, 2009). The $\mathrm{N}$-terminal domain of FtsZ, a guanosine triphosphate hydrolase (GTPase), is responsible for polymerization through the head-to-tail interaction between the guanosine triphosphate (GTP) binding pocket and the C-terminal T7 loop (de Boer et al., 1992; Egan and Vollmer, 2013). FtsZ polymerization is highly regulated by several proteins, which are classified as positive or negative regulators depending on their effects. Positive regulators of FtsZ are crucial for tethering FtsZ polymers to the membrane and encouraging the formation of stable lateral interactions to assemble a mature Z-ring (Huang et al., 2013). Negative regulators of FtsZ have a critical role in positioning FtsZ at the prospective site of division and for sustaining Z-ring dynamics by regulating the process of FtsZ polymer assembly and disassembly (Huang et al., 2013).

Zaps, which are FtsZ positive regulators, include ZapA, ZapB, ZapC, and ZapD and they are structurally characterized (Ebersbach et al., 2008; Low et al., 2004; Ortiz et al., 2015; Roach et al., 2016). ZapA is well known as a positive regulator of Z-ring formation, which can bind to FtsZ at the globular core directly (Galli and Gerdes, 2012). ZapC also interacts with FtsZ at the globular core and increases Z-ring stability (Huang et al., 2013). Recently, the crystal structure of ZapC from E. coli showed that ZapC is a monomer composed of two domains and it binds the FtsZ globular core rather than C-terminal tail (Schumacher et al., 2016). ZapD deletion in cells resulted in no significant change in cell viability, however, decreased viability was observed in the polymerization-defective FtsZ84 (Ts) cells, 
suggesting that ZapD is a positive regulator of FtsZ protofilament formation (Durand-Heredia et al., 2012; Huang et al., 2013). ZapD was shown to directly bind the C-terminal tail region of FtsZ as a positive regulator (Durand-Heredia et al., 2012).

Despite extensive previous studies on the architecture of the Z-ring, the molecular mechanism of how FtsZ protofilaments are assembled to form a mature Z-ring by Zap proteins is still poorly understood. Towards such an understanding, we sought to characterize the structure and function of ZapD to elucidate how ZapD promotes the assembly of FtsZ protofilaments and acts as a positive regulator of Z-ring formation. Here, we report the crystal structures of two ZapD proteins from S. typhimurium and $E$. coli, which share $89 \%$ amino-acid sequence identity. Several conserved residues are clustered on the concave surface of the EcZapD dimer, which is the suggested FtsZ binding site. The opposite surface of the StZapD or EcZapD dimer is rich in conserved positively charged Arg residues. The structural and biochemical studies on ZapD from $S$. typhimurium and $E$. coli suggest that the two FtsZ binding sites of StZapD and EcZapD dimers might be responsible for binding to two FtsZ protofilaments to hold them together.

\section{MATERIALS AND METHODS}

\section{Cloning and protein preparation}

The S. typhimurium $\mathrm{ZapD}_{1-247}$ gene was amplified with polymerase chain reaction (PCR) from its genomic DNA. The forward and reverse oligonucleotide primers were $5^{\prime}-\mathrm{C}$ G CTA TAT GGA TCC C GAA AAC CTG TAT TIT CAG GGC ATG CAC ACC CAG GTC CTA TTT G-3' and 5'-G CTA ATT CTC GAG TTA GCA ACA GGC CAG TTC AAA AT-3', respectively. The bases in bold represent the BamHI and Xhol restriction enzyme cleavage sites, and underlined sequences indicate the additional Tobacco Etch Virus (TEV) protease recognition site. The amplified DNA was digested using $B a m H I$ and $X h o l$, and then introduced into BamHI/Xhol digested pRSFDuet-1 (Novagen) expression vector.

Methods for expression and purification of EcZapD protein (residues 2-247) are similar to previously published methods (Son and Lee, 2015). The EcZapD WT and mutants (residues 1-247, E8A, E22R, H140R, or R176A) were cloned into a pHisparallel2 vector (Sheffield et al., 1999). Constructs of EcZapD WT and EcZapD mutants with an N-terminal His-tag followed by TEV protease cleavage site (ENLYFQG) were expressed in E. coli strain BL21(DE3)-pLysS cells as a host, while StZapD with an N-terminal His-tag followed by TEV protease cleavage site was expressed in E. coli strain BL21(DE3) cells as a host. The cells were treated with $0.5 \mathrm{mM}$ isopropyl- $\beta-\mathrm{D}-1$ thiogalactopyranoside (IPTG) to induce protein expression at $20^{\circ} \mathrm{C}$ for $20 \mathrm{~h}$. Cells were lysed by passage through a Microfluidizer (Microfluidics, USA) in $20 \mathrm{mM}$ Tris- $\mathrm{HCl} \mathrm{pH} \mathrm{7.9,500} \mathrm{mM}$ $\mathrm{NaCl}, 5 \mathrm{mM}$ imidazole, and $1 \mathrm{mM}$ phenylmethylsulfonyl fluoride (PMSF). The protein was purified using a Ni-sepharose affinity column, and was further purified by size exclusion chromatography (HiLoad 16/600 Superdex 75 prep grade, GE Healthcare) and stored in small aliquots at $-80^{\circ} \mathrm{C}$.

EcFtsZ peptides (GST-FtsZ $367-383$, GST-FtsZ $Z_{367-383}$ K380D, GST-Fts $Z_{372-383 \text {, and GST-FtsZ }}$ G67-379) were fused to an Nterminal GST tag followed by a TEV protease cleavage site. The GST-FtsZ $Z_{367-383}$ construct was amplified by PCR. The forward and reverse primers used to amplify the GST-FtsZ $Z_{367-383}$ construct were 5'-G CTA TAT GGA TCC AAA GAG CCG GAT TAT CTG GAT-3' and 5'-G CTA ATT CTC GAG TTA ATC AGC TTG CTT ACG-3', respectively. The bases in bold repre- sent BamHI and $\mathrm{Xhol}$ restriction enzyme cleavage sites. Amplified DNA was digested with $\mathrm{BamHI}$ and $\mathrm{XhOl}$ and was then inserted into the BamHI/Xhol-digested expression vector pGST-parallel2 (Sheffield et al., 1999). The plasmids were transformed into E. coli BL21(DE3) cells (Invitrogen), and cells harboring the plasmids were grown at $37^{\circ} \mathrm{C}$ until the optical density (at $600 \mathrm{~nm}$ ) reached 0.7-1.0 in Luria Bertani (LB) broth containing $100 \mu \mathrm{g} / \mathrm{ml}$ ampicillin. Then, $0.5 \mathrm{mM}$ IPTG was used to induce protein expression in the cells, after which the cells were incubated for $3 \mathrm{~h}$ at $37^{\circ} \mathrm{C}$. Cells were harvested by centrifugation at $5,000 \mathrm{rpm}$ at $4^{\circ} \mathrm{C}$ for $20 \mathrm{~min}$, and the pellet was resuspended in ice-cold lysis buffer $\mathrm{A}(20 \mathrm{mM}$ Tris- $\mathrm{HCl} \mathrm{pH} 8.0$ and $200 \mathrm{mM} \mathrm{NaCl}$ ) containing $1 \mathrm{mM}$ PMSF. The cells were lysed using microfluidizer (Microfluidics, USA), and the lysed cells were centrifuged at 12,000 rpm (Vision V506CA rotor) at $4^{\circ} \mathrm{C}$ for $30 \mathrm{~min}$ to separate the supernatant and cell debris. The supernatant was applied to a glutathione-Sepharose column (GE Healthcare) pre-equilibrated with lysis buffer. Initially, the column was washed extensively with lysis buffer $A$, after which the protein was eluted with elution buffer $(150 \mathrm{mM}$ Tris- $\mathrm{HCl} \mathrm{pH}$ 8.0 and $15 \mathrm{mM}$ reduced glutathione). Further purification was performed by gel filtration on a HiLoad 16/600 Superdex 75 prep-grade column (GE Healthcare), which was equilibrated with buffer $A$.

Crystallization, structure determination, and refinement The crystallization method and the data processing parameters for EcZapD (residues 2-247) has been published elsewhere (Son and Lee, 2015). The structure of EcZapD was solved by the molecular replacement method using the DUF1342 model from Vibrio parahaemolyticus (unknown function, PDB ID: 2OEZ) as the probe. Crystals of the StZapD (residues 1-247) were grown at $293 \mathrm{~K}$ using the sitting drop method by mixing 1 $\mu \mathrm{l}$ of the StZapD solution at $12 \mathrm{mg} / \mathrm{ml}$ in $20 \mathrm{mM}$ Tris- $\mathrm{HCl}(\mathrm{pH}$ 8.0), $200 \mathrm{mM} \mathrm{NaCl}$, and $1 \mathrm{mM}$ dithiothreitol (DTT) with $1 \mu \mathrm{l}$ of the reservoir solution consisting of $1.1 \mathrm{M}$ sodium malonate, 100 mM HEPES ( $\mathrm{pH} 7.5)$, and 0.5\%(v/v) Jeffamin ED-2001 (pH7.0). Data sets were collected at $100 \mathrm{~K}$ in $1^{\circ}$ oscillations at the BL38B1 beamline of SPring-8. Crystals of StZapD diffracted to a resolution of $2.6 \AA$ (Table 1), and the diffraction data were processed and scaled using the HKL2000 software package (Otwinowski and Minor, 1997). The structure was solved using the molecular replacement method using the DUF1342 model as a probe. The crystal belonged to space group $\mathrm{P}_{3} 22$ with unit cell parameters of $a=84.2 \AA, b=84.2 \AA$, and $c=150.4 \AA$. A cross-rotational search followed by a translational search was performed using the PHASER program (McCoy et al., 2007). Subsequent manual model building was performed using the COOT program (Emsley and Cowtan, 2004) and restrained refinement was carried out using the REFMAC5 program (Murshudov et al., 1997). Several rounds of model building, simulated annealing, positional refinement, and individual Bfactor refinement were performed using the COOT and REFMAC5 programs. As the test data for the calculation of $R_{\text {free }}$ $5 \%$ of the data were randomly set aside (Brunger, 1992). The coordinates and structure factors for StZapD and EcZapD have been deposited in the Protein Data Bank under accession numbers 5GNP and 5IMJ, respectively.

\section{Size exclusion chromatography with multi-angle light} scattering (SEC-MALS)

SEC-MALS experiments for EcZapD and StZapD were performed using a FPLC system (GE Healthcare) connected to a Wyatt MiniDAWN TREOS MALS instrument and a Wyatt Op- 
tilab rEX differential refractometer. A Superdex 200 10/300 GL (GE Healthcare) gel-filtration column pre-equilibrated with buffer A was normalized using ovalbumin protein. Proteins were injected $(1 \mathrm{mg})$ at a flow rate of $0.4 \mathrm{ml} / \mathrm{min}$. Data were analyzed using the Zimm model for static light-scattering data fitting and graphed using EASI graph with a UV peak in the ASTRA V software (Wyatt).

Bio-layer interferometry (BLI) measurement

BLI measurements were carried out to demonstrate binding between EcZapD (WT or mutants) and EcFtsZ (GST-FtsZ

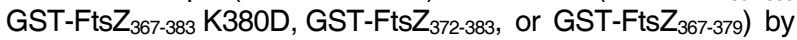
using a BLItz system (ForteBio, Menlo Park, CA). EcFtsZ pro-

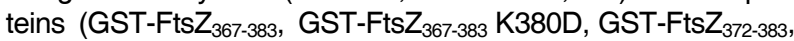
or GST-FtsZ $367-379$ ) were immobilized on an AR2G biosensor chip surface using amine coupling in $20 \mathrm{mM}$ sodium acetate (pH 4.0). The surface was activated by a 5-min immersion of $\mathrm{N}$ hydroxysuccinimide (NHS)/ethyl(dimethyl-aminopropyl) carbodiimide (EDC). Subsequently, the AR2G biosensor chip was blocked by a 5-min immersion of $1 \mathrm{M}$ ethanolamine. The surface was equilibrated with buffer $A$. To determine whether interactions occur between EcZapD (WT, E22R, and H140R)

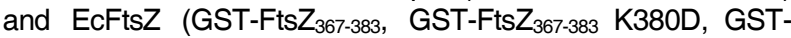
FtsZ $Z_{372-383}$, or GST-FtsZ $Z_{367-379}$ ), the prepared AR2G biosensor chip was dipped in $35 \mu \mathrm{M}$ protein solution in running buffer. Associations between EcZapD (WT, E22R, and H140R) and EcFtsZ (GST-FtsZ 383, or GST-FtsZ $367-379$ ) were measured for $150 \mathrm{~s}$, and dissociations were measured for $150 \mathrm{~s}$.

\section{RESULTS AND DISCUSSION}

Structure determination of EcZapD and StZapD

We have determined the crystal structures of EcZapD (residues 2-247) and StZapD (1-247) by the molecular replacement method using the DUF1342 model from $V$. parahaemolyticus (unknown function, PDB ID: 2OEZ) as the probe. To facilitate crystallization, the $\mathrm{N}$-terminus of EcZapD (residue number 1) was truncated. Two monomers of EcZapD belong to the space group $P 6_{4}$, with unit cell parameters of $a=b=109.5 \AA$, and $c=$ 106.7 $\AA$. The model of EcZapD has been refined to crystallographic $R_{\text {work }}$ and $R_{\text {free }}$ values of $22.7 \%$ and $28.3 \%$ for the $35.4-$ 3.1 A data (Table 1). The refined EcZapD model has one dimer in an asymmetric unit with 492 residues, 5 sulfate ions, and 41 water molecules, respectively. Ramachandran plot analysis for the model showed that $86.3 \%$ and $10.2 \%$ of the non-glycine residues were in the most favored and allowed regions, and $3.5 \%$ of them were in disallowed regions (Table 1 ). The outlier residues are located in regions of fairly poor density.

One monomers of StZapD belong to the space group $P 6_{3} 22$, with unit cell parameters of $\mathrm{a}=\mathrm{b}=84.2 \AA$, and $\mathrm{c}=150.0 \AA$. The model of StZapD has been refined to crystallographic $R_{\text {work }}$ and $R_{\text {free }}$ values of $24.8 \%$ and $27.7 \%$ for the $50-2.8 \AA$ data (Table 1). The refined StZapD model has one monomer in an asymmetric unit with 245 residues, 1 malonate ion, and 36 water molecules. Ramachandran plot analysis for the model showed that $95.9 \%$ and $3.7 \%$ of the non-glycine residues were in the most favored and allowed regions, and $0.4 \%$ of them were in disallowed regions (Table 1). The outlier residues are located in regions of fairly poor density.

\section{Overall structure of StZapD and its oligomeric state in} solution

The StZapD monomer is composed of two domains; the $\mathrm{N}$ -
Table 1. Statistics for data collection and refinement

\begin{tabular}{lc}
\hline \multicolumn{1}{c}{ Data set } & StZapD \\
\hline A. crystal parameters & \\
X-ray source & BL38B1 \\
X-ray wavelength $(\AA)$ & 1.0000 \\
Space group & $\mathrm{P} 6_{3} 22$ \\
Unit cell parameters $(\AA)$ & $\mathrm{a}=84.2$ \\
& $\mathrm{~b}=84.2$ \\
& $\mathrm{C}=150.0$ \\
Resolution range $(\AA))$ & $50-2.8$ \\
Total / unique reflections & $107,772 / 8,289$ \\
Completeness $(\%)$ & $99.7(100.0)^{\mathrm{a}}$ \\
Average $/ / \sigma(I)$ & $48.6(5.9)^{\mathrm{a}}$ \\
$R_{\text {rim }}{ }^{b}(\%)$ & $11.3(62.4)^{\mathrm{a}}$ \\
$R_{\text {pim }}{ }^{c}(\%)$ & $3.2(16.3)^{\mathrm{a}}$ \\
$C^{C} C_{1 / 2}{ }^{d}(\%)$ & $99.7(93.7)^{\mathrm{a}}$
\end{tabular}

\section{B. Model refinement statistics}

$\begin{array}{lcc}\text { Resolution range }(\AA) & 50-2.8 & 35.4-3.1 \\ R_{\text {work }} / R_{\text {free }}{ }^{e}(\%) & 24.8 / 27.7 & 22.7 / 28.3 \\ \text { Number / average } B \text {-factor }\left(\AA^{2}\right) & & \\ \quad \begin{array}{l}\text { Protein nonhydrogen atoms } \\ \text { Water oxygen atoms }\end{array} & 1,983 / 83.0 & 3,968 / 95.28 \\ \quad \text { Malonate } & 36 / 72.7 & 41 / 83.07 \\ \text { R.m.s. deviations from ideal } & 1 / 100 & \\ \quad \text { Bond lengths }(\AA) & & \\ \text { Bond angles }\left({ }^{\circ}\right) & 0.005 & 0.007 \\ \text { Protein-geometry analysis } & 0.927 & 1.208 \\ \quad \text { Ramachandran favored }(\%) & & \\ \text { Ramachandran allowed }(\%) & 35.9 & 86.3 \\ \text { Ramachandran outliers }(\%) & 0.4 & 10.2 \\ \end{array}$

${ }^{a}$ Values in parentheses refer to the highest resolution shell (2.85-2.80 $\left.\AA\right)$ ${ }^{b} \mathrm{R}_{\text {rim }}=\Sigma_{\text {hk }\{}\{N(h k l) /[N(h k)-1]\}^{1 / 2} \Sigma_{i}\left|l_{i}(h k l)-<l(h k l)>\right| / \Sigma_{\text {hk }} \Sigma_{i} l_{i}(h k)$. The redundancy-independent merging $R$ factor gives the precision of individual intensity (Diederichs and Karplus, 1997).

$\left.{ }^{c} R_{\text {pim }}=\Sigma_{\text {nkl }\{1} /[N(h k l)-1]\right\}^{1 / 2} \Sigma_{i}\left|l_{i}(h k l)-</(h k)>\right| / \Sigma_{h k l} \Sigma_{i} l_{i}(h k l)$. The precision indicating merging $R$ factor describes the precision of the averaged intensity (Weiss, 2001)

${ }^{d} \mathrm{CC}_{1 / 2}$ is the correlation coefficient of the mean intensities between two random half-sets of data (Diederichs and Karplus, 2013).

${ }^{e} R=\Sigma_{\text {hkl }}|| F_{\text {obs }}|-| F_{\text {calc }}|| / \Sigma_{\text {hkl }}\left|F_{\text {obs }}\right|$, where $R_{\text {free }}$ was calculated for a randomly chosen $5 \%$ of reflections, which were not used for structure refinement and $R_{\text {work }}$ was calculated for the remaining.

The data processing parameters for EcZapD have been described elsewhere with the reference (Son and Lee, 2015)

terminal $\alpha$-helices domain (NTD) and the C-terminal $\beta$-sheet domain (CTD) (Figs. 1 and 2). The NTD consists of eight $\alpha$ helices, while the CTD consists of seven $\beta$ strands (Figs. 1 and $2)$. The CTD is composed of two antiparallel $\beta$-sheets $(\beta 1-\beta 4-$ $\beta 7-\beta 2$ and $\beta 5-\beta 6-\beta 3$, respectively) facing each other (Fig. 1A). Interestingly, the $\mathrm{N}$-terminal $\beta 1$ is part of the $\mathrm{C}$-terminal $\beta$-sheet. The asymmetric crystallization unit contains one monomer, and its dimeric assembly with approximate dimensions of $90 \AA \times 60$ 
A
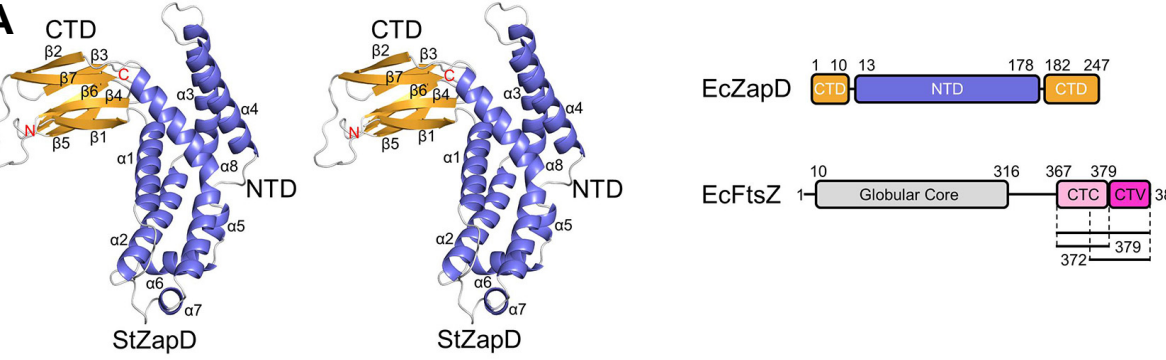

B

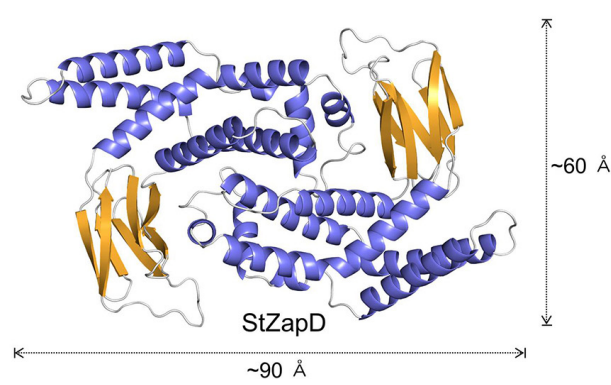

C

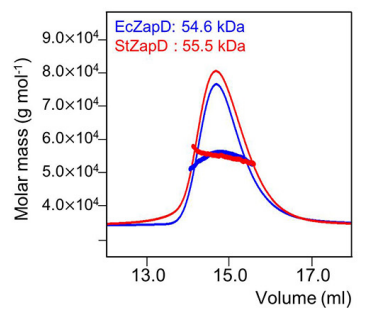

Fig. 1. Overall structure of StZapD and oligomeric state in solution. (A) Stereo ribbon diagram of monomer structure of StZapD. The NTD (Nterminal domain) and CTD (Cterminal domain) are shown in light blue and gold, respectively. Loops are drawn in gray. Right panel indicates domain architectures of EcZapD and EcFtsZ (Huang et al., 2016). EcFtsZ constructs used in biochemical analysis are shown in bold line. C-terminal constant region and $\mathrm{C}$-terminal variable region are denoted as CTC and CTV, respectively. (B) Ribbon diagram of StZapD dimer structure. (C) The molecular weights of EcZapD and StZapD in solution were analyzed by SECMALS. The thick lines represent the measured molecular weight.

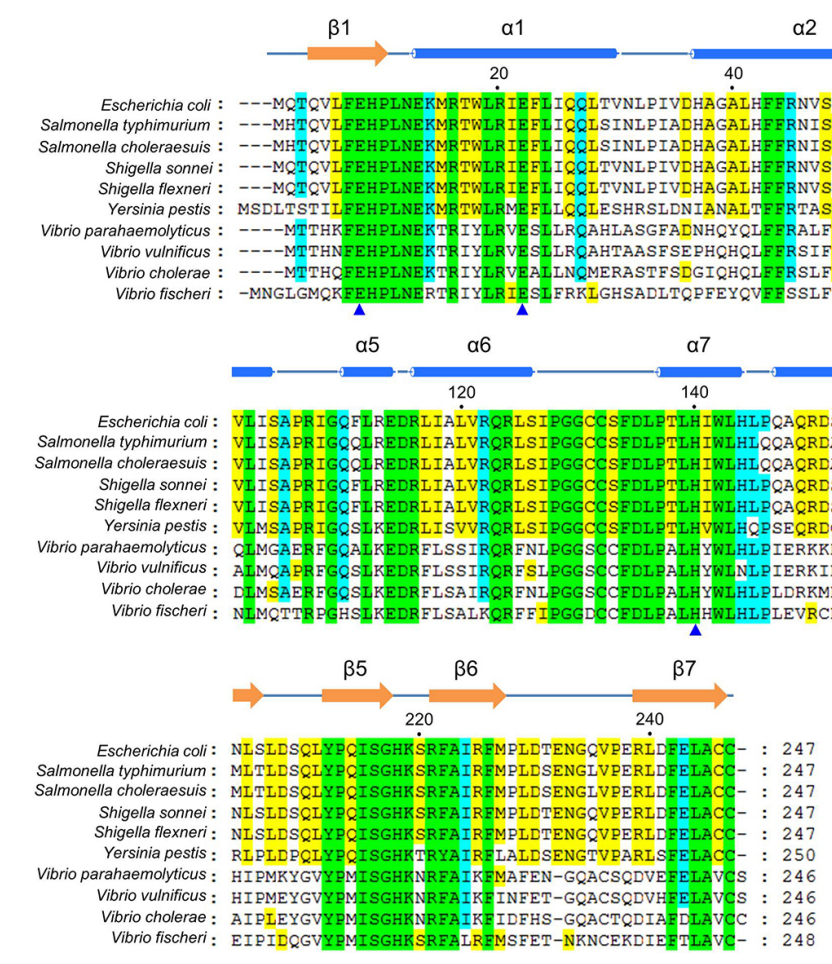

Fig. 2. Sequence alignments of StZapD with other ZapD proteins. Multi-alignment of E. coli (UniProtKB/Swiss-Prot accession number J7QWY2) against ZapD from S. typhimurium ZapD (UniProtKB/Swiss-Prot accession number P67693), Salmonella choleraesuis (UniProtKB/Swiss-Prot accession number Q57TB7), Shigella sonnei (UniProtKB/Swiss-Prot accession number Q3Z5Q7), Shigella flexneri (UniProtKB/Swiss-Prot accession number Q83MF4), Yersinia pestis (UniProtKB/Swiss-Prot accession number Q8ZBH9), Vibrio parahaemolyticus (UniProtKB/Swiss-Prot accession number Q87LT3), Vibrio vulnificus (UniProtKB/Swiss-Prot accession number P67695), Vibrio cholerae serotype 01 (UniProtKB/Swiss-Prot accession number P67694), and Vibrio fischeri (UniProtKB/Swiss-Prot accession number Q5E2R1). Secondary structure elements were assigned by PyMOL (The PyMOL Molecular Graphics System, http://www.pymol.org) and every twentieth residue is marked by a black dot. Strictly (100\%) and semi-conserved residues ( $80 \%$ and $60 \%)$ are highlighted in green, cyan, and yellow, respectively. Arrows and cylinders above the sequences denote $\alpha$-helices and $\beta$-strands, respectively. Blue triangles indicate the mutation sites for EcZapDEcFtsZ binding study. 
A

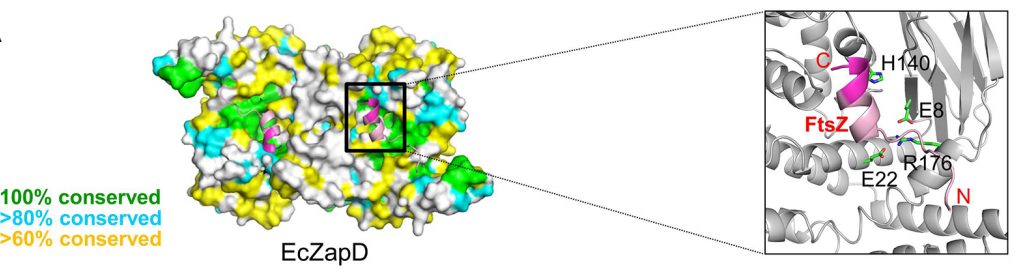

B
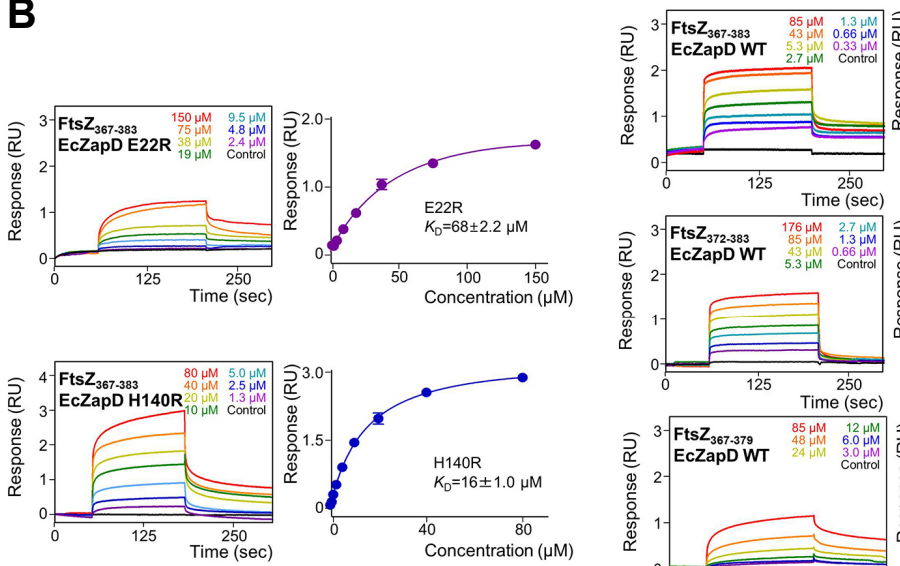
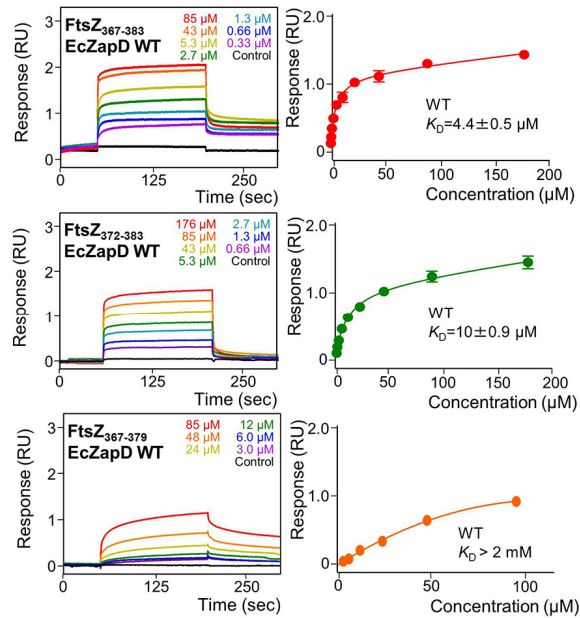
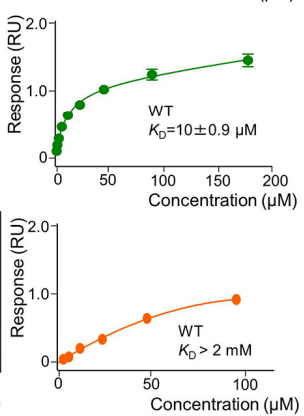

Fig. 3. Modeled structure of EcZapD in complex with FtsZ peptides (residues 367-383). (A) Modeled structure of EcZapD-FtsZ complex. Sequence conservation was mapped onto the surface of the EcZapD dimer. The conserved residues are shown in green $(100 \%)$, cyan (greater than $80 \%$ ), and yellow (greater than $60 \%$ ). CTC and CTV regions are shown in light pink and magenta colors, respectively (Huang et al., 2016). (B) Left two panels indicate representative BLI binding sensorgrams of GST-FtsZ $367-383$ with EcZapD mutants (E22R and H140R). Right three panels indicate representative BLI binding sensorgrams of EcZapD WT with GST-FtsZ ${ }_{367-383}$, GST-Fts $_{372-383}$, and GST-FtsZ $Z_{367-379}$, respectively. The experiments were repeated three times. Each concentration of analytes is shown in a different color.
$\AA$ × $30 \AA$ presents twofold symmetry (Fig. 1B). To unveil overall structural similarities with other known structures from PDB, a search using the DALI program was performed (Holm and Rosenstrom, 2010). It showed several structurally similar proteins including (i) cell division protein ZapD from E. coli (5DKO, Z-score 30) (Roach et al., 2016), (ii) the DUF1342 protein (unknown function) from $V$. parahaemolyticus (2OEZ, Z-score 27), and (iii) Integron cassette protein from $V$. paracholerae (3JRT, Z-score 8.2) (Sureshan et al., 2013). When we compared our StZapD refined structure with the high Z-score structures (PDB codes 5DKO, 2OEZ, and 3JRT), the r.m.s. deviations were 0.9 $\AA(240 \mathrm{C} \alpha), 2.0 \AA(240 \mathrm{C} \alpha)$, and $5.6 \AA(120 \mathrm{C} \alpha)$ for $\mathrm{C} \alpha$ atom pairs, respectively.

The solvent-accessible surface area buried at the interface between the two monomers in the dimeric unit of StZapD was calculated by PISA (Krissinel and Henrick, 2007), to be $\sim 1871$ $\AA^{2}$ (13\% of the monomer surface area), suggesting that StZapD may exist in solution as the dimer that is present in the crystal. To further analyze the oligomeric states of StZapD and EcZapD in solution, we measured the molecular weight of StZapD using SEC-MALS. The molecular masses of StZapD and EcZapD were 55.5 and $54.6 \mathrm{kDa}$, close to the theoretical molecular mass of dimeric StZapD (56.9 kDa) and EcZapD (56.8 kDa) in solution, respectively (Fig. $1 \mathrm{C}$ ), indicating that the functional oligomeric state of StZapD and EcZapD in solution is dimer. It is interesting to note that other structurally characterized Zap proteins (ZapA, ZapB, and ZapC) also exist as dimer in solution, even though ZapA exists in a dimer-tetramer equilibrium in concentration-dependent manner (Ebersbach et al., 2008; Low et al., 2004; Ortiz et al., 2015).

The binding activity of EcZapD to the C-terminal peptide of FtsZ

The C-terminal peptide of FtsZ consists of highly conserved residues and interacts with diverse FtsZ-interacting proteins, including FtsZ, ZipA, SepF, EzrA, and ZapD (Huang et al., 2013; Son and Lee, 2013). A previous report showed that the C-terminal peptide of FtsZ could be divided into two parts-the C-terminal constant region (CTC, ${ }^{367} \mathrm{KEPDYLDIPAFLR}^{379}$ in EcZapD) consisting of conserved residues, and a C-terminal variable region (CTV, ${ }^{380} \mathrm{KQAD}^{383}$ in EcZapD) consisting of sequences diverse in length and composition (Huang et al., 2013). To confirm the predicted function of EcZapD as an FtsZ binding protein, we attempted to measure the catalytic activity of FtsZ binding by bio-layer interferometry (BLI) experiments. $\mathrm{BLI}$ assays were performed by attaching C-terminal FtsZ peptides from E. coli (residues 367-383, 372-383, or 367-379) with GST tags (GST-FtsZ $367-383$, GST-FtsZ ${ }_{372-383}$, or GST-FtsZ $Z_{367-379}$ ) on an AR2G biosensor chip to check whether EcZapD (WT or mutants) contains peptide binding activity (Fig. 3). To ensure that the EcZapD proteins did not bind to the GST protein, but to the C-terminal FtsZ peptide specifically, a control chip containing GST only without the C-terminal FtsZ peptide was utilized. Indeed, EcZapD bound tightly to the GST-FtsZ ${ }_{367-383}$ but not to the GST protein only (Figs. $3 A$ and $3 B$ ), indicating that the interaction between EcZapD and the C-terminal FtsZ peptide is specific. Thus, the FtsZ binding activity suggests that EcZapD is a direct modulator of FtsZ protofilaments. To check whether the $\mathrm{N}$-terminal part of the CTC region is essential for EcZapD binding, we attempted to remove the five $\mathrm{N}$-terminal residues (residues 367-371) of the CTC region for an EcZapD binding study. The binding affinities of EcZapD with EcFtsZ proteins (residues 367-383 and 372-383) were $4.4 \pm 0.5$ and $10 \pm 0.9$ $\mu \mathrm{M}$, respectively, suggesting that residues $372-383$ of FtsZ are sufficient for its binding to EcZapD (Fig. 3B). In addition, to check whether the CTV region is essential for EcZapD binding, we removed the CTV part (residues 380-383) and measured its binding affinity with EcZapD. The binding affinity was significantly lower $\left(\mathrm{K}_{\mathrm{d}}>2 \mathrm{mM}\right)$ than those of other EcFtsZ constructs (residues $367-383$ or 372-383), suggesting that the CTV region 
A

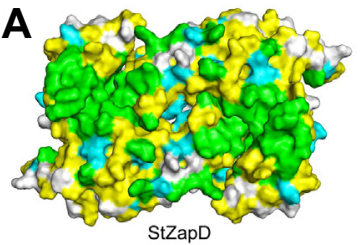

\section{B}
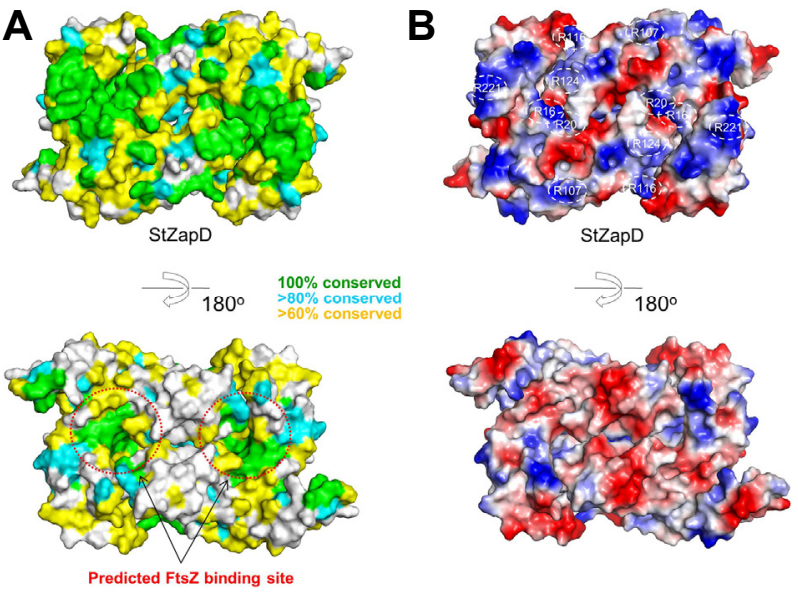

Fig. 4. Surface representation of the sequence conservation and electrostatic potential of StZapD. (A) Sequence conservation mapped onto the surface of the StZapD dimer. The conserved residues are green $(100 \%)$, cyan (greater than $80 \%$ ), and yellow (greater than $60 \%$ ). The predicted FtsZ binding sites are indicated as red dotted circles. (B) Surface representation and electrostatic potential rotated $180^{\circ}$ around the indicated axis in comparison to the upper figure are shown below. The positions of conserved positively charged residues are indicated.

of FtsZ, ${ }^{380} \mathrm{KQAD}^{383}$, is essential for binding to EcZapD (Fig. 3B). In recent study, the importance of K380 of FtsZ in the CTV region was reported (Huang et al., 2016). Thus, we performed another BLItz assay between EcFtsZ (367-383) K380D mutant and EcZapD and measured its binding affinity $\left(\mathrm{K}_{d}=4.7 \pm 0.5\right.$ $\mu \mathrm{M})$ (Supplementary Fig. S1A). It showed similar binding affinity with EcFtsZ WT (367-383) (Fig. 3B), suggesting that the mutation of K380D in EcFtsZ (367-383) is not enough to abolish the interaction with EcZapD.

\section{Putative FtsZ binding sites on EcZapD and modeled} structure of EcZapD $\mathrm{D}_{2-247}-\mathrm{FtsZ}_{367-383}$ complex

When the molecular surface of EcZapD was drawn, two deep clefts between two monomers were found on the concave surface of EcZapD (Fig. 3). The calculated volumes of each cavity using the CASTp server (Dundas et al., 2006) were $166 \AA^{3}$ and $98 \AA^{3}$, respectively. Along this cleft, several conserved residues are clustered, including Glu8, Glu22, His140, and Arg176 (Fig. $3 A)$. This cleft appeared to be the site of FtsZ binding, thus we tried to solve the crystal structure of $\mathrm{EcZapD}_{2-247}-\mathrm{FtsZ}_{367-383}$ complex. However, we could not produce the crystal of Ec$Z_{2 a p D}{ }_{2-247}-F t s Z_{367-383}$ complex despite of various screening trials. Thus, using the FlexPepDock server (London et al., 2011), we have modeled the complex of $\mathrm{EcZapD}_{2-247}$ with $\mathrm{FtsZ}_{367-383}$ to identify the $\mathrm{FtsZ}_{367-383}$ binding site on $\mathrm{EcZapD}_{2-247}(\mathrm{Fig}$. 3A). As expected, the deep clefts between the two monomers, which were suggested to be the FtsZ binding site, were indeed responsible for Fts $Z_{367-383}$ binding. Along with the Fts $Z_{367-383}$ binding site, there were two strictly conserved residues (Glu22 and His140). Therefore, to validate this model, EcZapD mutants (E22R and H140R) were tested to determine if the mutations weaken the FtsZ binding affinity for EcZapD. Indeed, mutations of Glu22 and His140 to Arg led to slightly lower binding $\left(K_{d}=68\right.$ $\pm 2.2 \mu \mathrm{M}$ and $16 \pm 1 \mu \mathrm{M}$, respectively) than that with WT EcZapD $(4.4 \pm 0.5 \mu \mathrm{M})$, suggesting the contributions of the two conserved residues to the binding between EcZapD and EcFtsZ (Fig. 3B). We could not measure the binding affinities with the E8A and R176A mutants of $\mathrm{FtsZ}_{367-383}$, because these proteins were expressed in insoluble forms (Supplementary Fig S1B). Based on these results, we speculated that both Glu22 and His 140 contribute to the binding of EcZapD to FtsZ directly or indirectly. Although much remains to be learned about the structural architecture of FtsZ-ZapD complex and the mechanism of FtsZ protofilaments stabilization by EcZapD, the results and model presented here provide one foothold for furthering such an understanding.

Note: Supplementary information is available on the Molecules and Cells website (www.molcells.org).

\section{ACKNOWLEDGMENTS}

The authors thank the staff of beamline BL44XU at SPring-8, Japan for their assistance during the $\mathrm{X}$-ray experiments and $\mathrm{Mr}$. Jung Min $\mathrm{Ha}$ for their assistance with protein purification and crystallization experiments. This study was supported by a grant from the National Research Foundation (NRF) of Korea funded by the Korean government (2015R1A5A1008958 and 2015R1C1A1A01054842), a grant from the Korea CCS R\&D Center (KCRC; 2014M1A8A1049296), and a grant from Institute for Basic Science (IBS-R021-D1) to H.H.L.

\section{REFERENCES}

Adams, D.W., and Errington, J. (2009). Bacterial cell division: assembly, maintenance and disassembly of the $Z$ ring. Nat. Rev. Microbiol. 7, 642-653.

Brunger, A.T. (1992). Free R value: a novel statistical quantity for assessing the accuracy of crystal structures. Nature 355 , 472475

de Boer, P., Crossley, R., and Rothfield, L. (1992). The essential bacterial cell-division protein FtsZ is a GTPase. Nature 359, 254256.

Diederichs, K., and Karplus, P.A. (1997). Improved R-factors for diffraction data analysis in macromolecular crystallography. Nat. Struct. Biol. 4, 269-275.

Diederichs, K., and Karplus, P.A. (2013). Better models by discarding data? Acta Crystallogr. D Biol. Crystallogr. 69, 12151222.

Dundas, J., Ouyang, Z., Tseng, J., Binkowski, A., Turpaz, Y., and Liang, J. (2006). CASTp: computed atlas of surface topography of proteins with structural and topographical mapping of functionally annotated residues. Nucleic Acids Res. 34, W116-118.

Durand-Heredia, J., Rivkin, E., Fan, G., Morales, J., and Janakiraman, A. (2012). Identification of ZapD as a cell division factor that promotes the assembly of FtsZ in Escherichia coli. J. Bacteriol. 194, 3189-3198.

Ebersbach, G., Galli, E., Moller-Jensen, J., Lowe, J., and Gerdes, K (2008). Novel coiled-coil cell division factor ZapB stimulates Z ring assembly and cell division. Mol. Microbiol. 68, 720-735.

Egan, A.J., and Vollmer, W. (2013). The physiology of bacterial cell division. Ann. N Y Acad. Sci. 1277, 8-28.

Emsley, P., and Cowtan, K. (2004). Coot: model-building tools for molecular graphics. Acta Crystallogr. D Biol. Crystallogr. 60, 2126-2132.

Erickson, H.P. (2009). Modeling the physics of FtsZ assembly and force generation. Proc. Natl. Acad. Sci. USA 106, 9238-9243.

Galli, E., and Gerdes, K. (2012). FtsZ-ZapA-ZapB interactome of Escherichia coli. J. Bacteriol. 194, 292-302.

Hale, C.A., and de Boer, P.A. (1997). Direct binding of FtsZ to ZipA, an essential component of the septal ring structure that mediates cell division in E. coli. Cell 88, 175-185.

Holm, L., and Rosenstrom, P. (2010). Dali server: conservation mapping in 3D. Nucleic Acids Res. 38, W545-549.

Huang, K.H., Durand-Heredia, J., and Janakiraman, A. (2013). FtsZ ring stability: of bundles, tubules, crosslinks, and curves. J. Bacteriol. 195, 1859-1868. 
Huang, K.H., Mychack, A., Tchorzewski, L., and Janakiraman, A. (2016). Characterization of the FtsZ C-Terminal Variable (CTV). region in Z-Ring assembly and interaction with the Z-Ring stabilizer ZapD in E.coli cytokinesis. PLoS One 11, e0153337.

Krissinel, E., and Henrick, K. (2007). Inference of macromolecular assemblies from crystalline state. J. Mol. Biol. 372, 774-797.

London, N., Raveh, B., Cohen, E., Fathi, G. and Schueler-Furman, O. (2011). Rosetta FlexPepDock web server--high resolution modeling of peptide-protein interactions. Nucleic Acids Res. 39, W249-253.

Low, H.H., Moncrieffe, M.C., and Lowe, J. (2004). The crystal structure of ZapA and its modulation of FtsZ polymerisation. J. Mol. Biol. 341, 839-852.

Lowe, J., and van den Ent, F. (2001). Conserved sequence motif at the C-terminus of the bacterial cell-division protein FtsA. Biochimie 83, 117-120.

Ma, X., Sun, Q., Wang, R., Singh, G., Jonietz, E.L., and Margolin, W. (1997). Interactions between heterologous FtsA and FtsZ proteins at the FtsZ ring. J. Bacteriol. 179, 6788-6797.

Margolin, W. (2000). Themes and variations in prokaryotic cell division. FEMS Microbiol. Rev. 24, 531-548.

McCoy, A.J., Grosse-Kunstleve, R.W., Adams, P.D., Winn, M.D., Storoni, L.C., and Read, R.J. (2007). Phaser crystallographic software. J. Appl. Crystallogr. 40, 658-674.

Murshudov, G.N., Vagin, A.A., and Dodson, E.J. (1997). Refinement of macromolecular structures by the maximum-likelihood method. Acta Crystallogr. D Biol. Crystallogr. 53, 240-255.

Ortiz, C., Kureisaite-Ciziene, D., Schmitz, F., McLaughlin, S.H., Vicente, M., and Lowe, J. (2015). Crystal structure of the Z-ring associated cell division protein ZapC from Escherichia coli. FEBS Lett. 589, 3822-3828.

Osawa, M., Anderson, D.E., and Erickson, H.P. (2009). Curved FtsZ protofilaments generate bending forces on liposome membranes. EMBO J. 28, 3476-3484.
Otwinowski, Z., and Minor, W. (1997). Processing of X-ray diffraction data collected in oscillation mode. Macromol. Crystallogr. Part A 276, 307-326.

Pichoff, S., and Lutkenhaus, J. (2002). Unique and overlapping roles for ZipA and FtsA in septal ring assembly in Escherichia coli. EMBO J. 21, 685-693.

Roach, E.J., Wroblewski, C., Seidel, L., Berezuk, A.M., Brewer, D., Kimber, M.S., and Khursigara, C.M. (2016). Structure and mutational analyses of Escherichia coli ZapD reveal charged residues involved in FtsZ filament bundling. J. Bacteriol. 198, 1683-1693.

Schumacher, M.A., Zeng, W., Huang, K.H., Tchorzewski, L., and Janakiraman, A. (2016). Structural and functional analyses reveal insights into the molecular properties of the Escherichia coli Z ring stabilizing protein, ZapC. J. Biol. Chem. 291, 24852498.

Sheffield, P., Garrard, S., and Derewenda, Z. (1999). Overcoming expression and purification problems of RhoGDI using a family of "parallel" expression vectors. Protein Exp. Purif. 15, 34-39.

Son, S.H., and Lee, H.H. (2013). The N-terminal domain of EzrA binds to the $\mathrm{C}$ terminus of FtsZ to inhibit Staphylococcus aureus FtsZ polymerization. Biochem. Biophys. Res. Commun. 433, 108-114.

Son, S.H., and Lee, H.H. (2015). Crystallization and preliminary Xray crystallographic analysis of Z-ring-associated protein (ZapD). from Escherichia coli. Acta Crystallogr. F Struct. Biol. Commun. 71, 194-198.

Sureshan, V., Deshpande, C.N., Boucher, Y., Koenig, J.E., Stokes, H.W., Harrop, S.J., Curmi, P.M., and Mabbutt, B.C. (2013) Integron gene cassettes: a repository of novel protein folds with distinct interaction sites. PLoS One 8, e52934.

Weiss, M.S. (2001). Global indicators of X-ray data quality. J. Appl. Cryst. 34, 130-135. 